\title{
Discerning the painter's hand: machine learning on surface topography
}

\author{
F. Ji ${ }^{1 \dagger}$, M. S. McMaster ${ }^{2 \dagger}$, S. Schwab ${ }^{2 \dagger}$, G. Singh ${ }^{2 \dagger}$, L. N. Smith ${ }^{3 \dagger}$, S. Adhikari ${ }^{2}$, M. O'Dwyer ${ }^{2}$, F. Sayed ${ }^{5}$, A. Ingrisano ${ }^{6}$, \\ D. Yoder ${ }^{4}$, E. S. Bolman ${ }^{3}$, I. T. Martin ${ }^{2+}$, M. Hinczewski ${ }^{2+}$ and K. D. Singer ${ }^{2 *}+$ (D)
}

\begin{abstract}
Attribution of paintings is a critical problem in art history. This study extends machine learning analysis to surface topography of painted works. A controlled study of positive attribution was designed with paintings produced by a class of art students. The paintings were scanned using a chromatic confocal optical profilometer to produce surface height data. The surface data were divided into virtual patches and used to train an ensemble of convolutional neural networks (CNNs) for attribution. Over a range of square patch sizes from 0.5 to $60 \mathrm{~mm}$, the resulting attribution was found to be 60-96\% accurate, and, when comparing regions of different color, was nearly twice as accurate as CNNs using color images of the paintings. Remarkably, short length scales, even as small as a bristle diameter, were the key to reliably distinguishing among artists. These results show promise for real-world attribution, particularly in the case of workshop practice.
\end{abstract}

Keywords: Topography, Machine intelligence, Convolutional neural networks (CNNs), Attribution

\section{Introduction}

Machine learning (ML) analysis for artwork is a budding methodology aimed at advancing connoisseurship, the primary method of determining the attribution of an artwork, among other applications involving artistic style. ML was successfully applied to images of paintings for tasks including detecting forgeries [1, 2], classifying digital collections [3, 4], and discerning an artist's style [5-8]. While many of these studies have applied ML to high-resolution photographic images of paintings, in this report, we use ML to analyze topographical data obtained by optical profilometry. Further, advancements in the resolution, speed, and availability of such non-contact profilometric measurements are growing alongside big-data methods that can handle the large

\footnotetext{
*Correspondence: kenneth.singer@case.edu

${ }^{\dagger}$ F. Ji, M. S. McMaster, S. Schwab, G. Singh and L. N. Smith contributed equally to this work

${ }^{\dagger}$ I. T. Martin, M. Hinczewski and K. D. Singer are senior project leads

2 Department of Physics, Case Western Reserve University, Cleveland, $\mathrm{OH}$

44106, United States

Full list of author information is available at the end of the article
}

datasets produced by these measurements. In paintings, surface topography reveals unintended stylistic elements embedded in the surface of the painting that may include the deposition and drying of the paint, patterns in the brushwork, physiological factors, and other aspects of the painting's creation.

A critical aspect of assessing attribution is understanding how members of artist-led workshops created works of art [9]. Many notable artists, including El Greco, Rembrandt, and Peter Paul Rubens, employed workshops, of varying sizes and structures, to meet market demands for their art. Connoisseurship has been widely successful at establishing a basis for workshop attribution in art historical studies. Additional information on the methods of connoisseurship in technical art history, and an overview of workshop practices is presented in the first section of Additional file 1. Practitioners of connoisseurship examine the visible stylistic elements of a composition, along with material elements, condition, and other clues about the fabrication process to build a historical understanding of the attribution of an artwork. Yet, many of the

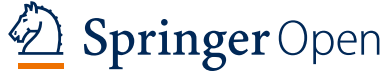

(c) The Author(s) 2021. Open Access This article is licensed under a Creative Commons Attribution 4.0 International License, which permits use, sharing, adaptation, distribution and reproduction in any medium or format, as long as you give appropriate credit to the original author(s) and the source, provide a link to the Creative Commons licence, and indicate if changes were made. The images or other third party material in this article are included in the article's Creative Commons licence, unless indicated otherwise in a credit line to the material. If material is not included in the article's Creative Commons licence and your intended use is not permitted by statutory regulation or exceeds the permitted use, you will need to obtain permission directly from the copyright holder. To view a copy of this licence, visit http://creativecommons.org/licenses/by/4.0/. The Creative Commons Public Domain Dedication waiver (http://creativeco mmons.org/publicdomain/zero/1.0/) applies to the data made available in this article, unless otherwise stated in a credit line to the data. 
specifics concerning workshop practice remain elusive. In the case of workshops, the various artists attempt to create a complete painting with a singular style, challenging the methods of connoisseurship. Further, the challenges of such attributions create conflict when the attribution is closely tied to the apparent value of objects in the art market. Hence, there is need for unbiased and quantitative methods to lend insight into disputed attributions of workshop paintings.

We hypothesize that significant unintended stylistic information exists in the 3D surface structure embedded by the painter during the painting process, and that this information can be captured through optical profilometry. In addition, differences in the artist's intrinsic and unintended style will be revealed by investigating patches at a much smaller length scale than any recognizable feature of the painting. By focusing on small features, we move away from intended stylistic information and allow for comparison of nearby regions within the same work of art. We further propose that a measurable unintended stylistic property that exists over small length-scales could be used to identify different hands in the same work of art; it could then be useful in attributing historic workshop paintings.

In this report, a controlled study of paintings commissioned from several artists that mimic certain workshop practices are interrogated for indicative stylistic information. The aim of this experiment is to explore the questions that (a) the brushstroke-produced high resolution profilometry data from a painting's surface contain stylistic information (i.e., painters leave behind a measurable "fingerprint"), and (b) the data are such that ML analysis can quantitatively distinguish among painters by the topographical information. Therefore, the experimental goal is to categorize small areas from the surface of paintings by their stylometric information, without the influence of purposeful stylistic choices (e.g., tools or materials) or factors regarding the subject of the painting. To this effect, a series of twelve paintings by four artists, and their associated topographical profiles, are subject to analysis to attribute the works and to ascertain the important properties involved in those attributions. Our use of identical materials and subject matter among the artists creates a stronger focus on individual stylistic components and reflects the properties and goals of workshop paintings. The methods and controls we employ here are suited to each individual task: namely, to isolate stylistic components, to test the efficacy of ML techniques on surface topography for proper attribution among several artists, to determine the length scales involved in the ML results, to compare with photographic ML, and to provide a basis for later studies on workshop paintings. We note, however, that the methods described here could find application elsewhere, such as forgery detection in contemporary art.

\section{Design and data analysis Designing a controlled experiment to study the so-called painter's hand}

Historical paintings have known and unknown variables that contribute to their physical states, including the materials used, the artist or artists' technique and style, and damage and restoration that have occurred over time. Each of these may contribute to the attribution of a painting. To ensure control over the stylistic and subjective content of the test set of paintings, we enlisted nine painting students from the Cleveland Institute of Art. Each artist created triplicate paintings of a fixed subject, a photograph of a water lily (Fig. 1). Each painting was created using the same materials (paint, canvas) and tools (paintbrushes) as described in the "Materials and methods" section below. In addition, the artists were instructed to treat the three versions as copies. To guide our investigation, four painting specialists (three art historians and a painting conservator) grouped the paintings by artist style using traditional connoisseurship, selecting the works of four of the nine artists for our investigation based on their stylistic similarity.

\section{Acquiring and preparing data from paintings}

The surface height information for each painting was collected by high resolution optical profilometry. Measurements were conducted over a $12 \times 15 \mathrm{~cm}$ region centered around the subject of the painting, with a spatial resolution of 50 microns, and a height repeatability of $200 \mathrm{~nm}$. Given that brush strokes and their associated features are on the scale of hundreds of microns, this was sufficient for capturing the fine brushstroke features of the painting's surface.

In preparation for the experiments, the height information is digitally split into small patches, the central objects of the investigation, as depicted in Fig. 1B. A typical patch size for these experiments is $1 \times 1 \mathrm{~cm}$, or $200 \times 200$ pixels, though we eventually explored a range of patch sizes from 10 pixels $(0.5 \mathrm{~mm})$ to 1200 pixels $(60 \mathrm{~mm})$. The effect of this is threefold. First, it eliminates the subjective information (the water lily figure) from the patches, since these patches are too small to individually contain indicatory information. Second, it gives a large enough set of data to enable using ML methods for each painter. For example, the 3 paintings will provide 540 patches at the $1 \times 1 \mathrm{~cm}$ patch size. Finally, we use ML to quantitatively attribute the individual patches of the painting. So, by reconstructing the original topography from the patches, we can visually represent regions of the surface with different quantitative attributions. This will be important 


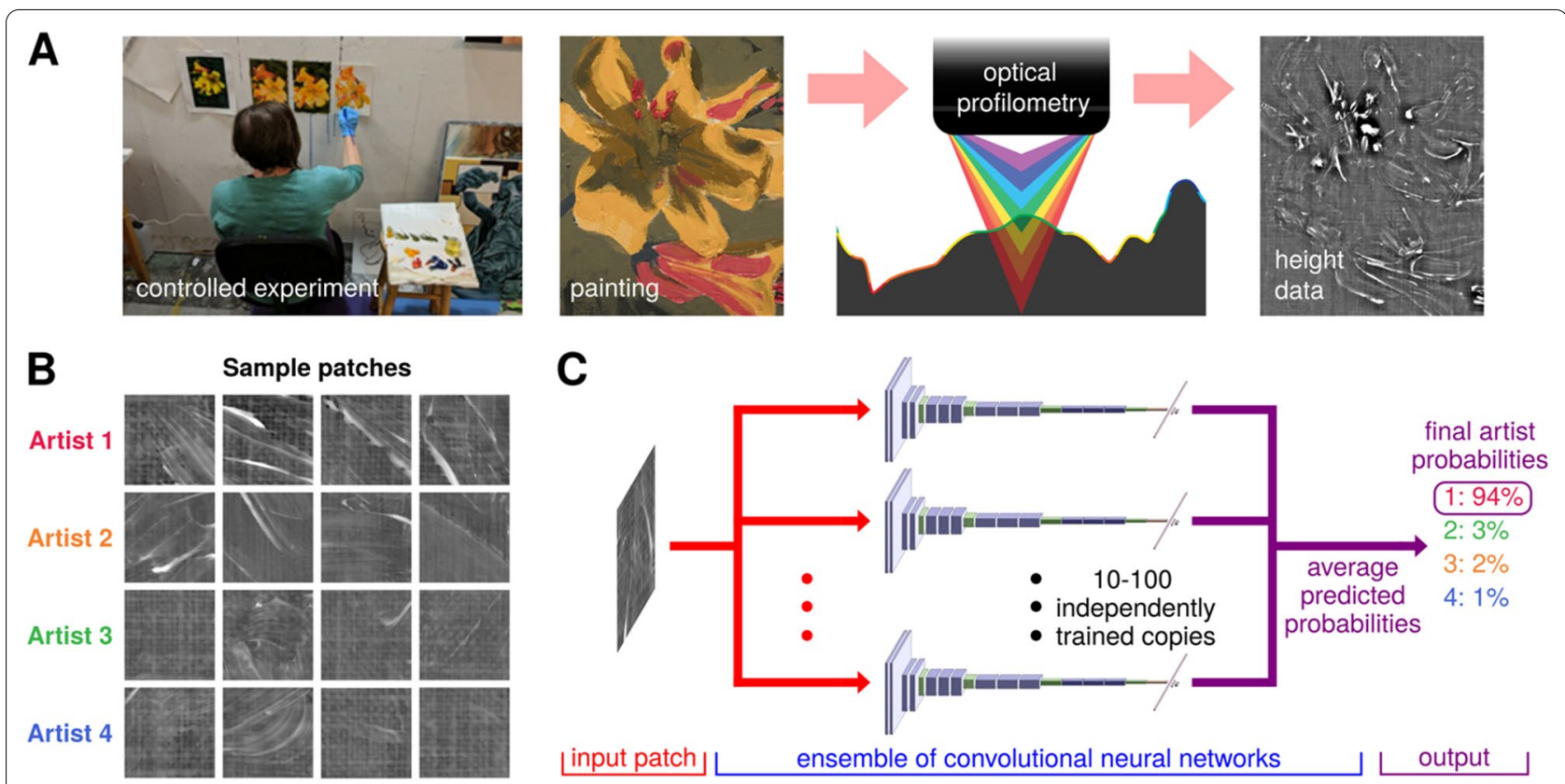

Fig. 1 A Overview of the data acquisition workflow. B Sample patches of height data from each artist; each patch is $200 \times 200$ pixels $(10 \times 10 \mathrm{~mm})$. C Schematic of the ML approach, which uses an ensemble of convolutional neural networks (CNNs) to assign artist attribution probabilities to each patch

for our future studies inspecting historical paintings, where different regions may represent the contributions of different hands, whether from different members of the workshop or later conservation attempts.

ML methods were then applied to the surface topographical information from the paintings to explore the following questions:

1. Is there enough information to differentiate among artists?

2. Which length scales contribute useful information?

3. How does topographical information compare to photographic data?

4. What machine learning methods provide the most accurate performance?

\section{Machine learning to reliably attribute patches of topographical data to individual artists}

Convolutional neural networks (CNNs) are a powerful and well-established method in computer vision tasks such as image classification $[10,11]$. They generally consist of three classes of layers: convolution, pooling, and fully connected layers [12] (see Additional file 1: Fig. S2). Convolution layers learn translation-invariant features from the data and pooling summarizes the learned features. The stacking of these layers helps to build a hierarchical representation of the data. Fully connected layers input these extracted features into a classifier and output image classes or labels. Identification using CNNs is ideal for signals-such as topographical data-that have local spatial correlations and translational invariance. However, training a deep $\mathrm{CNN}$ from scratch on a small dataset typically results in a problem known as over-fitting, where the network performs better on the training set, but often does not generalize well to unseen data. To avoid this, a common solution is transfer learning [13]: adapting a network that has been pretrained on a large dataset to a different but related task. For the case of CNNs pretrained on images, the initial layers perform general feature extraction, and hence are often applicable to a broad variety of image classification problems. The final fully connected layer (and sometimes several of its predecessors) is replaced and retrained for the problem of interest. This retraining of the network is fine-tuned in a block-wise manner, starting with tuning the last few layers, and then allowing further preceding layers to be trainable as well. In this work, the network we have used is an architecture called VGG-16 [14], which was pretrained on more than one million images in the ImageNet dataset [15].

This transfer learning procedure allows us the full functionality of a highly tuned deep CNN with specificity to our task of surface topography. In short, this CNN now is outfitted to take a small input patch of the painting and produce an output pertaining to attribution. The output 
of our network is a 4-D vector whose components correspond to the probability of attribution to one of the four artists in the experiment (Fig. 1C). Patches from two of the three paintings from each artist are used for training/ validation, with patches from the third painting reserved for testing. Because of the stochastic nature of the training procedure, involving presenting random minibatches from the training set over many epochs, the weights in the final network after training would be different if we were to repeat the whole procedure from the start. We take advantage of this stochasticity by creating ensembles of 10-100 different trained networks for each task we consider, using the mean of the probability vectors from the entire ensemble as the final prediction. We then calculate the overall accuracy as well as $\mathrm{F}_{1}$ scores, which is a measure of test accuracy for each artist. Such ensemble learning predictions in many cases outperform those of single networks [16]. Additional details of the network architecture, training, and fine-tuning procedure can be found in the "Material and methods" section and Additional file 1.

\section{Results and discussion}

\section{Artist attribution results}

The results of ensemble ML of 100 different trained networks for attribution using patches of side-length 200 pixels $(10 \mathrm{~mm})$ are shown in Fig. 2. Each patch is color coded according to the highest probability (most likely artist), with the opacity of the shading proportional to the magnitude of that probability (i.e., more transparent shadings correspond to more uncertain attributions). Out of 180 patches for each artist in the test painting, we found $12,0,2$, and 14 patches attributed incorrectly for artists 1 through 4, representing an overall accuracy of $96.1 \%$. This is remarkable as $25 \%$ accuracy is expected by random choice. Further, we find that most of the patches were attributed with high confidence (more opaque shading) for all four artists. The accuracy of ML prediction from the height data is remarkable, particularly given the similarity of the patches in terms of features distinguishable to the human eye (Fig. 1B), as well as its success in broad monochrome areas of the painted background.

\section{Exploring the effect of patch size on attribution accuracy}

The surprisingly accurate attribution of $10 \mathrm{~mm}$ patches leads to a natural question: how does the size of the patch affect the machine's ability to properly attribute? In other words, can we make the patch size smaller than $10 \mathrm{~mm}$ and still reliably attribute the hand? Fig. 3 presents results for networks trained on patches with different side-lengths ranging from 10 pixels $(0.5 \mathrm{~mm})$ to 1200 pixels $(60 \mathrm{~mm})$. The predictions are quantified in terms of overall accuracy for all four artists (solid thick curve) and individual artist $\mathrm{F}_{1}$ score (thin colored curves). We also calculated precision and recall; results are shown in Additional file 1: Fig. S3. To check the self-consistency of the predictions, we conducted repeat training/testing trials at each patch size (details in the Additional file 1). The data points and error bars in Fig. 3 represent the mean and standard deviation for those trials.

The accuracy exhibits a broad plateau around 95\% for patches between 100 and 300 pixels (5 and $15 \mathrm{~mm}$ ). Below 100 pixels there is a gradual drop-off in accuracy, as each individual patch contains fewer of the distinctive features that facilitate attribution. The $\mathrm{F}_{1}$ scores allow us to separate out the network performance for each artist. Consistent with the results in Fig. 2, the attribution is generally better for artists 2 and 3 versus 1 and 4 across patch sizes less than 300 pixels $(15 \mathrm{~mm})$. Nonetheless, the $\mathrm{F}_{1}$ scores for all artists are above $90 \%$ near the optimal patch size (around 200 pixels or $10 \mathrm{~mm}$ ).

On the other end of the patch size spectrum, the ML approach faces a different challenge. The size of training sets becomes quite small, even though each individual patch contains many informative features. The singlenetwork accuracy drops off quickly for patch sizes above $300(15 \mathrm{~mm})$ pixels, decreasing to about $75 \%$ at the largest sizes.

\section{Predictions using single-pixel information versus spatial correlations}

One of the hallmarks of CNNs is their ability to harness spatial correlations at various scales in an input image in order to make a prediction. However, there is also information present at the single pixel level since each artist's height data will have a characteristic distribution relative to the mean. The probability densities for these distributions are shown in Fig. 4, calculated from the two paintings in the training sets of each artist. The height distributions are all single-peaked and similar in width, except for Artist 1, who exhibits a broader tail at heights below the mean than the others. In order to determine how important spatial correlations are, we can compare the $\mathrm{CNN}$ results to an alternative attribution method that is blind to the correlations: maximum likelihood estimation (MLE). For a given patch in the testing set, we calculate the total likelihood for the height values of every pixel in the patch belonging to each of the four distributions in Fig. 4. Attribution of the patch is assigned to the artist with the highest likelihood. The predictive accuracy of the MLE approach versus patch size is shown as a dashed line in Fig. 3. We expect MLE to perform the best at the largest patch sizes, since each patch then gives a larger sampling of the height distribution, and hence is easier to assign. Indeed, at the patch size of 1200 pixels $(60 \mathrm{~mm})$, representing nearly a fifth of the area of a single 


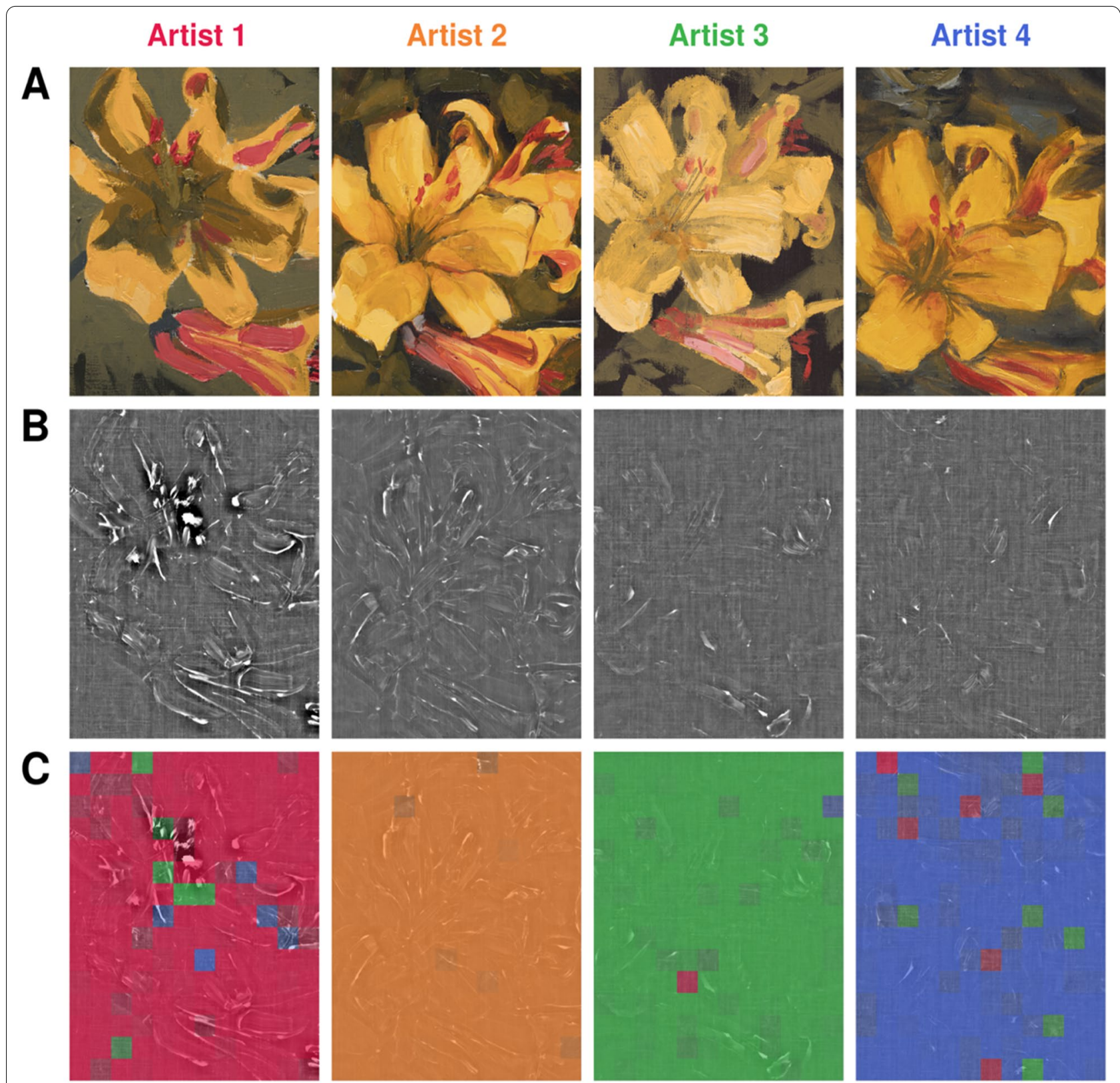

Fig. 2 Each artist (artists 1-4) created three paintings, one of which was reserved for testing the trained ML algorithm. These test paintings are shown for all four artists as $\mathbf{A}$ high-resolution photographs (all paintings used in the study are presented in Additional file 1: Fig. S1), B height data, shaded in grayscale from low (darker) to high (lighter). C Attribution results of the ensemble ML predictions on height data. Color shadings are overlayed on the grayscale image from row B corresponding to the most likely artist for each patch of side-length 200 pixels (10 mm; 1: red, 2: orange, 3: green, 4: blue). More opaque shades of color indicate greater predictive confidence (larger attribution probabilities). The overall accuracy of the patch attribution is $96.1 \%$

painting, the MLE accuracy approaches 70\%, comparable to the CNN accuracy. In this limit the size of the training set is likely too small for the $\mathrm{CNN}$ to effectively learn correlation features. As the patch size decreases, the gap between the CNN and MLE performance grows dramatically. In the range of $100-300$ pixels $(5-15 \mathrm{~mm})$ where the CNN performs optimally ( 95\%), the MLE accuracy is only around $40 \%$. These small patches are an insufficient sample of the distribution to make accurate attributions based on single pixel height data alone. Clearly the $\mathrm{CNN}$ is taking advantage of spatial correlations in the surface heights. This leads to a natural next question: 


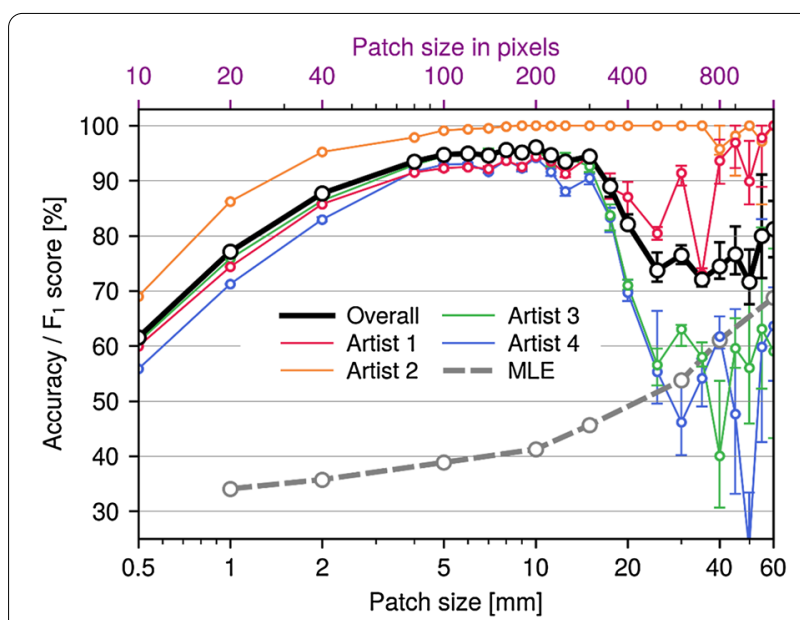

Fig. 3 Patch size versus two measures of ML performance: overall accuracy (thick black curve) and $F_{1}$ scores for each artist (thin colored curves). The best results occur for patches in the range 100-300 pixels $(\sim 5-15 \mathrm{~mm})$. The overall accuracy decreases when patch size is smaller than 100 pixels (due to lack of information in each patch) and when patch size is larger than 300 pixels (because the size of the training data set decreases with increasing patch size). Error bars are standard deviations over different repetitions of ensemble training. For comparison, the maximum likelihood estimation (MLE) accuracy results based on the pixel height distributions are shown as a dashed curve

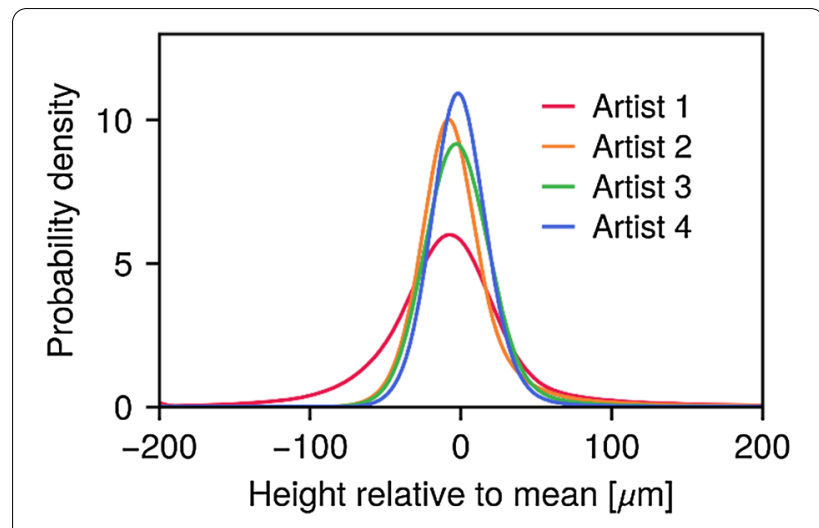

Fig. 4 Distributions of heights relative to the mean for each artist

what correlation length scales are involved in the attribution decision?

\section{Using empirical mode decomposition to determine the length-scales of the brushstroke topography}

In order to examine the spatial frequency (length) scales most important in the ML analysis, we employed a preprocessing technique used historically in timeseries signal analysis called empirical mode decomposition (EMD) [17], which has recently been extended into the spatial domain [18-20]. Its versatility is derived from its data-driven methodology, relying on unbiased techniques for filtering data into intrinsic mode functions (IMFs) that characterize the signal's innate frequency composition [21]. In our case, we have used a bi-directional multivariate EMD [22] to split our 3D reconstruction of each painting's complex surface structure into IMFs that characterize the various spatial scales present.

The first IMF contains the smallest length scale textures, and subsequent IMFs contain larger and larger features until the sifting procedure is halted and a residual is all that remains. This process is lossless in the sense that by adding each IMF and the resulting residual together, the entire signal is preserved $[17,21]$. It is also unbiased in the sense that when compared to standard Fourier analysis techniques, there are no spatial frequency boundaries to define, and no edge effects introduced from defining those boundaries.

By investigating each series of IMFs individually, we can estimate the length scale for each as follows. We use a standard 2D fast-Fourier transform on the IMF and calculate a weighted average frequency for the modes. The length scale is the inverse of the average frequency and is plotted versus IMF number in Fig. 5B. Among the four artists, the typical scale increases from about $0.2 \mathrm{~mm}$ for IMF 1 to $0.8 \mathrm{~mm}$ for IMF 5. Figure 5A shows a sample patch and the corresponding IMFs, which illustrates the progressive coarsening for the higher numbered IMFs. To see how the length scale affects the attribution results, we repeated the CNN training using each IMF separately, rather than the height data. The resulting mean accuracies for each IMF using three different patch sizes are shown in Fig. 5C. Individual IMFs are by construction less informative than the full height data (which is a sum of all the IMFs), and hence we do not reach the $95 \%$ level of accuracy seen in the earlier $\mathrm{CNN}$ results. However, IMFs 1 and 2 (the smallest length scales) achieve accuracies of above $80 \%$ at patch size $10 \mathrm{~mm}$ (200 pixels). There is a drop-off in accuracy as we go to larger patch sizes (IMFs 3-5), indicating that the salient information used for attribution is present at length scales of $0.2-0.4 \mathrm{~mm}$. These are comparable to the dimensions of a single bristle in the types of brushes used by the artists, which were 0.25 and $0.65 \mathrm{~mm}$ respectively, as shown as dashed lines in Fig. 5B. This strongly suggests that the key to this attribution using height data lies at scales that are small enough to reflect the unintended (physiological) style of the artist. This result is consistent with the scaledependent ML results depicted Fig. 3, which indicate that below a patch size of $5 \mathrm{~mm}$, all accuracies are well-above that expected for random attribution (25\%). Remarkably, even at the scale of $0.5 \mathrm{~mm}$, that is, the scale of 1-2 bristle widths, ML was able to attribute to $60 \%$ accuracy. 


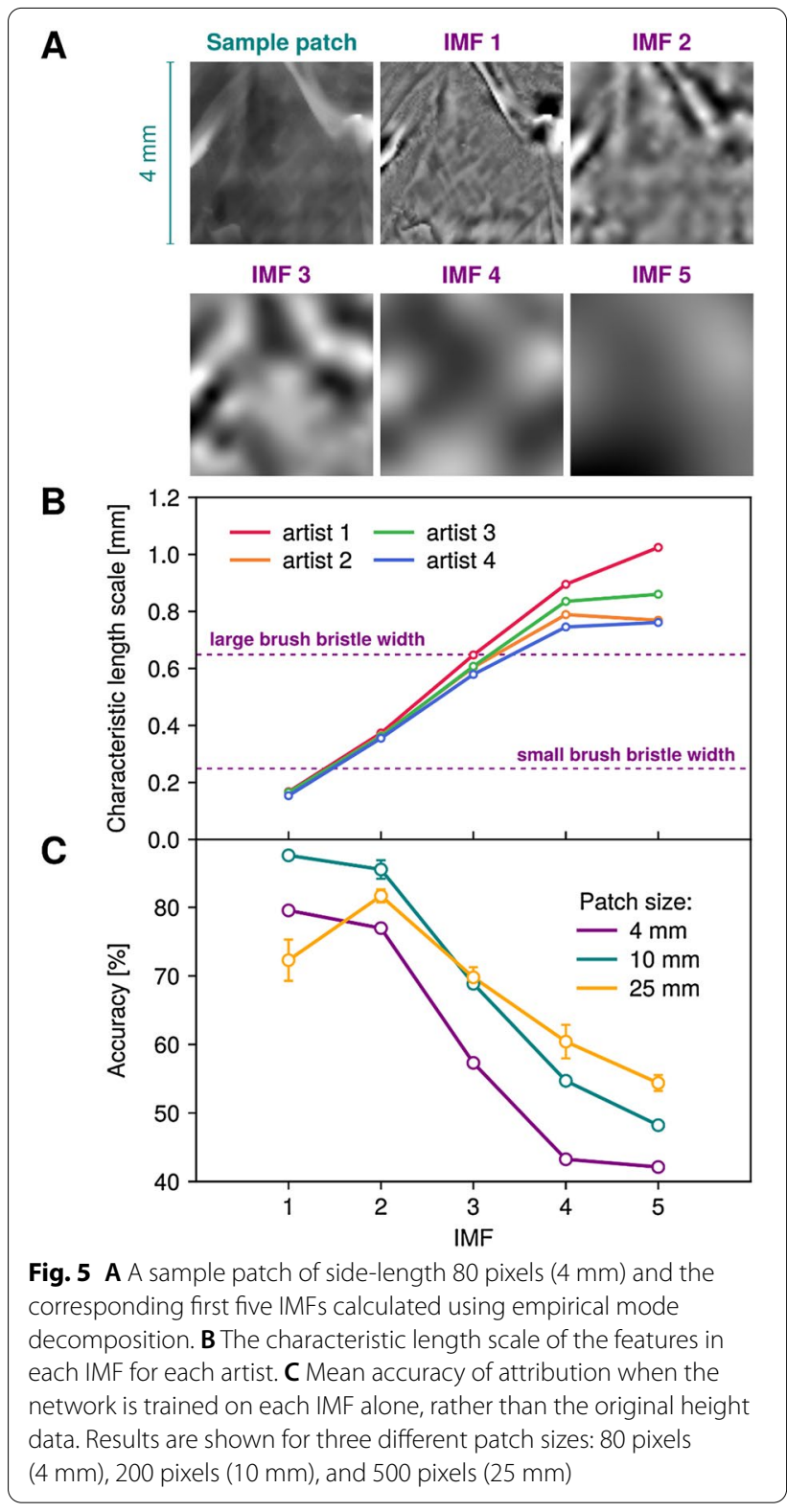

\section{Comparing topography versus photography when testing on data with novel characteristics}

Image recognition by ML is most often performed on photographic images of the subject depicted by arrays corresponding to the RGB channels of the entire image. The aim of this test is to determine how well CNNs perform at attributing the patches of the images depicted in row A of Fig. 2 as compared to the profilometry data. We were particularly interested in how well ML of the two types of data-photo and height-based-would perform if the testing set had novel colors and subject matter, absent in the training set. This approach better approximates the challenges of real-world attribution, where we would not necessarily have extensive well-attributed training data matching the palette and content of the regions of interest in a painting where the algorithm would be applied. To generate qualitatively distinct training and testing sets, we divided each painting into patches of side-length 100 pixels $(5 \mathrm{~mm})$ and then sorted the patches into three categories: background, foreground, and border depending on the color composition of each patch (see Fig. 6A for an example). Among our training set, $25 \%$ of the patches are assigned to background, $50 \%$ count as foreground, and the remaining $25 \%$ are border patches (Fig. 6B). The latter include regions of both background and foreground and were excluded from both training and testing to make it more challenging for the algorithm to generalize from one category to the other. The mostly dark green and black color palette and lack of defined subjects in the background distinguishes it from the foreground, which is dominated by the painted flower, with various shades of yellows and reds. Could a network trained on only background patches still accurately attribute foreground patches, or vice versa? The mean accuracy results are shown Fig. 6C, with the left two bars corresponding to training on the background, testing on the foreground, and the right two bars to the reverse scenario. Because the training sets are significantly smaller (and less representative of the test sets) than in our earlier analysis, we expect lower attribution accuracies. Despite this, networks trained on the height data (blue bars) perform reasonably well, achieving $60 \%$ accuracy when trained on background, and $80 \%$ when trained on foreground. (We note that the background training set is about half the size of the foreground one). In contrast, networks trained on the photo data did significantly worse (red bars), achieving $27 \%$ and $43 \%$ accuracies, respectively. Clearly, in this context the color and subject information in the photo data, which was likely the focus of the ML training, was a hindrance, since the test set confronted the network with novel colors and subject matter. In contrast, there is a significant, smallscale, stylistic component that is captured in the height data that is present whether the artist is painting the foreground or background, which is therefore harnessed for attribution.

\section{Conclusions}

We have described a controlled experiment using ML methods coupled with optical profilometry data to attribute painted works of art based on the topographical structure indicative of the artists' style as a tool for art connoisseurship. By dividing the paintings into patches significantly smaller than the painting, we have removed subject information as well as aspects of the artist's intended style. We are thereby able to 

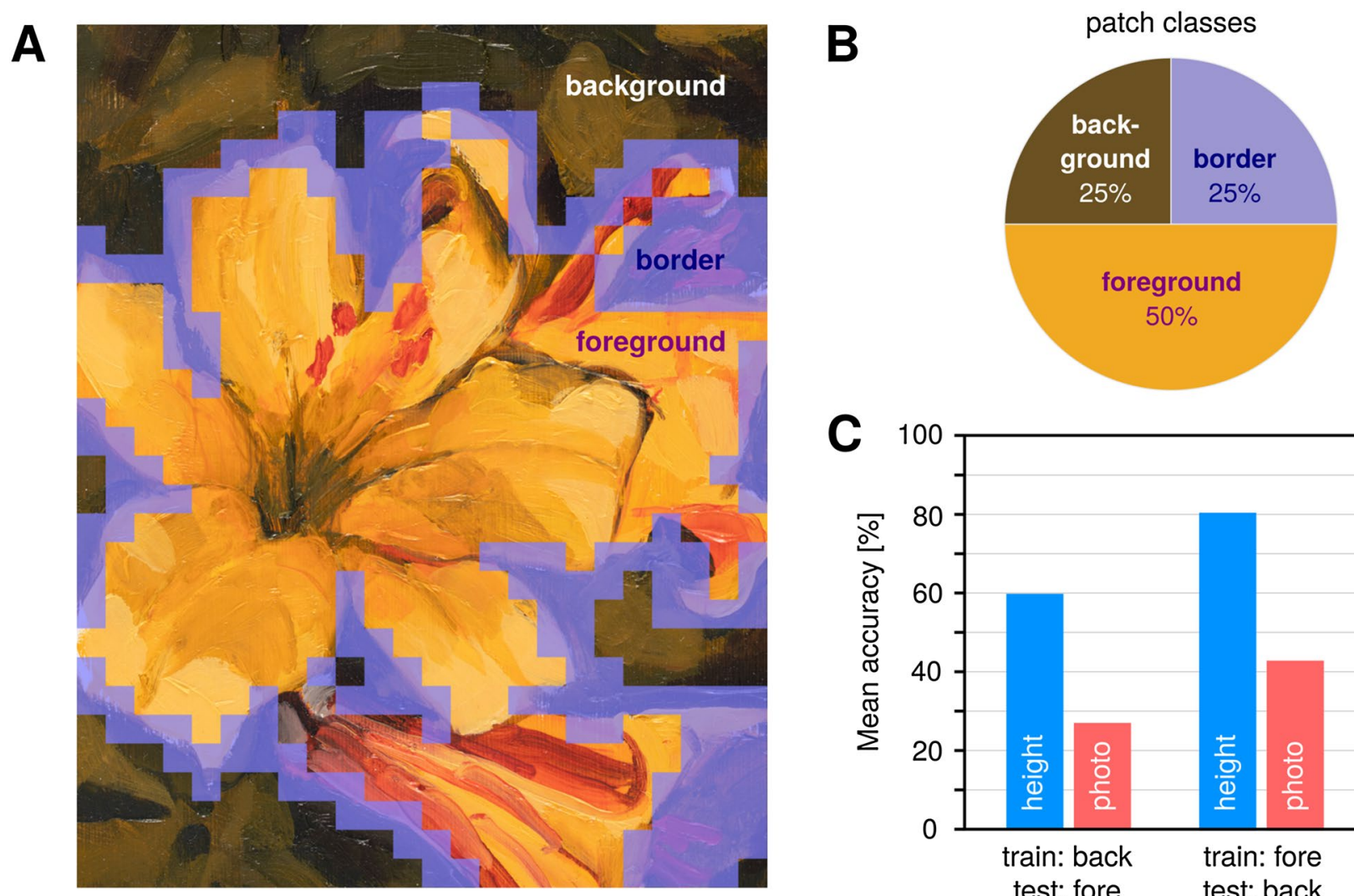

Fig. 6 A Example of a painting divided into background, foreground, and border (mixed background and foreground) patches of size $100 \times 100$ pixels $(5 \times 5 \mathrm{~mm})$. B Distribution of the three classes across all patches in the training set. $\mathbf{C}$ Results from training on background patches, testing on foreground patches and vice versa using both height data and high-resolution photo data

focus our study onto attribution using only unintended style components. We found outstanding attribution accuracy of over $90 \%$, in the best cases, using ensemble CNNs, and further, provided evidence using EMD alongside ML, that the smallest length scales, comparable to a small number of bristles, are most telling for the ML attribution process. This result suggests that our techniques are complementary to expert connoisseurship, which focus on longer length scales. Thus, surface topography expands the toolbox for attribution, conservation, forgery detection and cultural heritage preservation. Additionally, we found that profilometry data provides higher attribution accuracy than using photographs when the subject and color palettes of the training and testing data are significantly different. Our virtual patch analysis is appropriate for attributing workshop paintings. In our planned application to realworld workshop paintings, however, the toll of intervening years and conservation measures will challenge our techniques. Finally, given the fine height resolution of optical scanners, these methods may find application in other media.

\section{Materials and methods}

\section{Experimental design summary}

This study was designed to assess the potential usefulness of surface topography in the attribution of paintings. Sets of triplicate paintings were individually created by nine artists, all using the same tools, materials, and subject. The surface topographical information for each painting was recorded using optical profilometry. Then, ML technology was developed to attribute small square patches of the painting's topography to the artist who painted them. Lastly, data filtering software was used to separate the height data into IMFs, based on spatial frequency, to determine the length scales of interest. In this way, we demonstrate the usefulness of topographical information to attributing paintings, and further investigate the method.

\section{Materials}

The paintings were prepared using Winsor \& Newton Winton Oil Colors: Titanium White, Cadmium Red, Cadmium Yellow, French Ultramarine, and Burnt Umber (Blick), Utrecht Linseed Oil (Blick), on cut canvas paper 
from a Canson Foundation Canva-Paper Pad (Blick). The paint was applied with a classroom set of off-theshelf, Blick Scholastic Wonder White paintbrushes (sizes: Bright 8, Bright 4, Round 4), which were used at the artist's discretion.

\section{Preparation of the paintings}

In preparation for the OP measurements, the paintings were adhered to plexiglass donated by the Cleveland Museum of Art, using 3 M Super 77 Multipurpose Spray Adhesive. This was done to ensure the entire $12 \times 15 \mathrm{~cm}$ region of the painting stays within the $1.1 \mathrm{~cm}$ focal range of the optical profilometer for the duration of the measurement.

\section{Profilometry}

A NANOVEA ST400 Optical Profilometer was used to create a detailed height map of the surface using the Chromatic Light technique. Measurements were taken over an area of 12 by $15 \mathrm{~cm}$ with $50 \mu \mathrm{m}$ spatial resolution. Specifications for the P5 pen used for the measurement include a lateral resolution of $11 \mu \mathrm{m}$, a $10 \mathrm{~mm}$ z-range, and a height repeatability of $200 \mathrm{~nm}$ within that range. Three thousand and one $12 \mathrm{~mm}$ line scans were made across the samples, separated by $50 \mu \mathrm{m}$ in the y-direction. Measurements were aligned to the center of the painting, so a favorable ratio of subject and background could be collected.

\section{Data preparation}

As an initial preprocessing step to remove large scale height variations due to the curvature of the canvas, we estimated the canvas profile by applying a mean filter of radius 100 pixels to the height data. The resulting profile was subtracted from the raw height data. These relative height values were then mapped so that the range from -200 to 300 microns corresponds to the grayscale range $0-1$. The values were copied three times to make three identical channels in a 16-bit PNG image file, to meet the three-channel requirement of VGG-16. When we used CNN to analyze individual IMFs, we also copied each individual IMF three times. Then we divided each painting into patches of various sizes ranging from $0.5 \mathrm{~mm}(10$ pixels) to $60 \mathrm{~mm}$ (1200 pixels). We preprocessed each patch by subtracting the mean RGB values (103.939, $116.779,123.68)$, which were computed on all the training images in the ImageNet database, from each pixel. The default input size for VGG-16 was 224 pixels $\times 224$ pixels; we then resized all the patches to match this size.

Each artist created three paintings; one was chosen from each artist as the test painting, and the other two as training/validation paintings, with the ratio of the number of training and validation patches as 9:1. To examine how this choice of test versus training/validation paintings affects the network performance, we also looked at variations: out of the 81 possible combinations, we randomly selected 10 and provide the results in Additional file 1: Fig. S4. At the optimal patch size of 200 pixels, the average performance is over $90 \%$, comparable to the main text results.

\section{CNN architecture}

To perform transfer learning, we removed the three fully connected layers at the top of the pretrained VGG-16 network to get the base model. The convolutional base functions as a feature extractor and learns spatial hierarchies of input images. We then added a new average pooling layer (performed over a $2 \times 2$ pixel window, with stride 2), followed by a flatten layer with $25 \%$ dropout. Next is a fully connected layer with $25 \%$ dropout, where we used a ReLU (rectified linear unit) activation function and applied an L2 regularization penalty with regularization factor 0.001 . The final layer was a soft-max layer that performs 4-artist classification. The overall network architecture is shown in Additional file 1: Fig. S2. We used Adam as the optimizer. The learning rate was set to 0.001 and 0.0001 depending on the training phase.

During training, we first randomly initialized the network weights by training the model for 25 epochs with learning rate 0.001 and batch size 32 and saved the model with highest accuracy from this phase of training. Then we unfroze the last two blocks of the VGG-16 base to fine tune the weights. During this phase of training, we used a slower learning rate of 0.0001 and trained the network for 25 epochs with batch size 32 .

\section{Calculating ensemble accuracy and $F_{1}$ scores}

After training, the network outputs a vector of four probabilities for each patch, corresponding to the likelihood of attribution to one of the four artists in the experiment. We created ensembles of 10-20 different trained networks depending on the patch size (more information in Additional file 1) and calculated the mean probability vector, which we used to predict which patch is painted by which artist. After making predictions of all the patches on the testing painting, we calculated the overall accuracy and $F_{1}$ scores for each artist. To calculate the overall accuracy, we counted the number of correctly labeled patches and divided it by the total number of testing patches. For each individual artist, we counted the number of true positives (TP), false positives (FP), false negatives $(\mathrm{FN})$, and calculated the $\mathrm{F}_{1}$ scores according to $\mathrm{F} 1=\mathrm{TP} /[\mathrm{TP}+1 / 2(\mathrm{FP}+\mathrm{FN})]$. Additionally, we also calculated precision and recall for each artist. Precision measures the proportion of patches attributed to artist $\mathrm{X}$ that are actually from artist X; it is defined as Precision $=$ 
$\mathrm{TP} /(\mathrm{TP}+\mathrm{FP})$. Recall, on the other hand, measures the proportion of patches that are from artist $\mathrm{X}$ that are correctly attributed to artist $\mathrm{X}$; we calculated it according to Recall $=\mathrm{TP} /(\mathrm{TP}+\mathrm{FP})$.

\section{Attribution based on maximum likelihood estimation (MLE)}

To understand the role of spatial correlations in attribution accuracy, we also employed an MLE approach where these correlations are ignored. To implement this method, we first did kernel density estimation (using Silverman's rule for bandwidth [23] to obtain probability density functions $P_{i}(z)$ for the single-pixel heights $z$ relative to the mean for each of the four artists, $i=1, \ldots$ 4. These estimates, shown in Fig. 4, were based on combined data from the two paintings in the training set for each artist. For a given test patch, the log-likelihood that the $j$ th pixel's height value, $z_{j}$, belongs to the distribution of artist $i$ is: $\log P_{i}\left(z_{j}\right)$. The overall $\log$-likelihood that all pixels in the patch belong to artist $i$ is: $L_{i}=\Sigma_{j} \log P_{i}\left(z_{j}\right)$, where the sum runs over all height values in the patch. MLE assigns the attribution of the patch to the artist $i$ who has the largest $L_{i}$ value. The resulting mean accuracies are shown as a dashed line in Fig. 3.

\section{Abbreviations}

CNN: Convolutional neural network; EMD: Empirical mode decomposition; FN: False negative; FP: False positive; IMF: Intrinsic mode function; ML: Machine learning; MLE: Maximum likelihood estimation; TP: True positive.

\section{Supplementary Information}

The online version contains supplementary material available at https://doi. org/10.1186/s40494-021-00618-w.

Additional file 1. Further discussion on the methods of connoisseurship in technical art history, and an overview of workshop practices. Details of ensemble learning with figures additional figures. Figure S1. A test paintings from each artist. B, C Training paintings from each artist. Figure S2. Schematic of the convolutional neural network architecture. Figure S3. Patch size versus A precision and B recall for each artist. Figure S4. Mean accuracy versus patch size for the original choice of training set used in the main text (black curve), versus the mean results from a random selection of 10 alternative training sets (red curve), corresponding to different choices of which two out of three paintings from each artist to use for training.

\section{Acknowledgements}

We thank the following artists from the Cleveland Institute of Art for creating the paintings used in this study: Emily Imka, Jace Lee, Brandon Secrest, Maeve Billings (artists 1-4 respectively), Julia O'Brien, Jamie Cohen-Kiraly, D'nae Webb, Seneca Kuchar, and Nolan Meyer. We thank the Cleveland Museum of Art Photography Department for high-resolution photographs of the paintings. We thank NANOVEA for the illustration in Fig. 1A. We also acknowledge discussions and input by Prof. Catherine B. Scallen (Art History and Art) and Per Knutås of the Cleveland Museum of Art. We are grateful to Prof. Harsh Mathur and Nathaniel Tomczak for their careful reading of the manuscript and insightful comments.

\section{Authors' contributions}

Al, IM, MM, MO, FS, KS, LS, DY contributed to experimental design and data collection. SA, MH, FJ, MO, GS, SS contributed to machine learning calculations and data presentation. SS contributed to empirical mode decomposition. EB, LS, DY contributed to the technical art history context of the work. All authors contributed to writing and editing the manuscript. All authors read and approved the final manuscript.

\section{Funding}

This work was partially supported by a research award from Case Western Reserve University. The authors acknowledge the use of the Materials for Opto/electronics Research and Education (MORE) Center, a core facility at Case Western Reserve University (Ohio Third Frontier grant TECH 09-021). This work made use of the High Performance Computing Resource in the Core Facility for Advanced Research Computing at Case Western Reserve University for model training, testing, and deployment.

\section{Availability of data and materials}

The data and code associated with this manuscript is available in the GitHub repository, https://github.com/hincz-lab/machine-learning-for-art-attribution.

\section{Declarations}

\section{Competing interests}

The authors declare that they have no competing interests.

\section{Author details}

${ }^{1}$ Department of Biology, Case Western Reserve University, Cleveland, $\mathrm{OH}$ 44106, United States. ${ }^{2}$ Department of Physics, Case Western Reserve University, Cleveland, OH 44106, United States. ${ }^{3}$ Department of Art History and Art, Case Western Reserve University, Cleveland, $\mathrm{OH} 44106$, United States. ${ }^{4}$ Cleveland Museum of Art, Cleveland, OH 44106, United States. ${ }^{5}$ Hathaway Brown School, Shaker Heights, OH 44122, United States. ${ }^{6}$ Cleveland Institute of Art, Cleveland, $\mathrm{OH}$ 44106, United States.

Received: 19 August 2021 Accepted: 21 October 2021

Published online: 12 November 2021

\section{References}

1. Polatkan G, Jafarpour S, Brasoveanu A, Hughes S, Daubechies I. "Detection of forgery in paintings using supervised learning. IEEE. 2009. https://doi. org/10.1109/ICIP.2009.5413338.

2. Friedman T, Lurie DJ, Shalom A. "Authentication of Rembrandt's self-portraits through the use of facial aging analysis. Isr Med Assoc J. 2012;14:591.

3. Sabatelli M, Kestemont M, Daelemans W, Geurts P. Deep transfer learning for art classification problems. Proc Eur Conf Comput Vis Workshops. 2018. https://doi.org/10.1007/978-3-030-11012-3_48.

4. Conover DM, Delaney JK, Ricciardi P, Loew MH. Towards automatic registration of technical images of works of art. Int Soc Opt Photonics. 2011. https://doi.org/10.1117/12.872634.

5. Jafarpour S, Polatkan G, Brevdo E, Hughes S, Brasoveanu A, Daubechies I. Stylistic analysis of paintings using wavelets and machine learning. Eur Signal Process Conf. 2009;17:1220.

6. Hughes JM, Graham DJ, Rockmore DN. Quantification of artistic style through sparse coding analysis in the drawings of Pieter Bruegel the Elder. Proc Natl Acad Sci USA. 2010. https://doi.org/10.1073/pnas.09105 30107.

7. Abry P, Wendt H, Jaffard S. When Van Gogh meets Mandelbrot: multifractal classification of painting's texture. Signal Process. 2013. https://doi.org/ 10.1016/j.sigpro.2012.01.016.

8. Asmus J, Parfenov V. Characterization of Rembrandt self-portraits through digital-chiaroscuro statistics. J Cult Herit. 2019. https://doi.org/10.1016/j. culher.2018.12.005.

9. van de Wetering E. The search for the master's hand: an anachronism? (A summary). Int Kongr Kunstgesch. 1993;2:627-30. 
10. LeCun Y, Bottou L, Bengio Y, Haffner P. Gradient-based learning applied to document recognition. Proc IEEE. 1998;86(11):2278-324.

11. Krizhevsky A, Sutskever I, Hinton GE. Imagenet classification with deep convolutional neural networks. Adv Neural Inf Process Syst. 2012;25:1097-105.

12. Yamashita R, Nishio M, Do RKG, Togashi K. Convolutional neural networks: an overview and application in radiology. Insights Imaging. 2018;9(4):611-29.

13. Weiss DK, Khoshgoftaar TM, Wang D. A survey of transfer learning. J Big Data. 2016;3(1):1-40.

14. Simonyan K, Zisserman A. Very Deep Convolutional Networks for LargeScale Image Recognition. In: International Conference on Learning Representations; 2015. arXiv:1409.1556

15. Deng J, Dong W, Socher R, Li L-J, Li K, Fei-Fei L. ImageNet: a large-scale hierarchical image database. IEEE Conf Comput Vis Pattern Recognit. 2009;2009:248-55. https://doi.org/10.1109/CVPR.2009.5206848.

16. Sagi O, Rokach L. Ensemble learning: a survey. Wiley Interdiscip Rev Data Min Knowl Discov. 2018;8(4):e1249.

17. Huang NE, et al. The empirical mode decomposition and the Hilbert spectrum for nonlinear and non-stationary time series analysis. Proc $\mathrm{R}$ Soc Lond Ser A Math Phys Eng Sci. 1998;454(1971):903-95.
18. Hu J, Wang X, Qin H. Novel and efficient computation of Hilbert-Huang transform on surfaces. Comput Aided Geom Des. 2016;43:95-108.

19. El Hadji SD, Alexandre R, Boudraa AO. Two-dimensional curvaturebased analysis of intrinsic mode functions. IEEE Signal Process Lett. 2017:25(1):20-4.

20. Al-Baddai S, Al-Subari K, Tomé AM, Solé-Casals J, Lang EW. A green's function-based bi-dimensional empirical mode decomposition. Inf Sci. 2016;348:305-21.

21. Huang NE, Shen Z, Long SR. A new view of nonlinear water waves: the Hilbert spectrum. Annu Rev Fluid Mech. 1999;31(1):417-57.

22. Xia Y, Zhang B, Pei W, Mandic DP. Bidimensional multivariate empirical mode decomposition with applications in multi-scale image fusion. IEEE Access. 2019;7:114261-70.

23. Silverman BW. Density estimation for statistics and data analysis. London: Chapman \& Hall/CRC; 1986. p. 45.

\section{Publisher's Note}

Springer Nature remains neutral with regard to jurisdictional claims in published maps and institutional affiliations.

\section{Submit your manuscript to a SpringerOpen ${ }^{\circ}$ journal and benefit from:}

- Convenient online submission

- Rigorous peer review

- Open access: articles freely available online

- High visibility within the field

- Retaining the copyright to your article

Submit your next manuscript at $\boldsymbol{\nabla}$ springeropen.com 